\title{
A simple surface modification to generate atomically-flat and hydrophobic substrates for gliding assays with protein motors
}

\author{
Isadonna F. Tengganu ${ }^{\mathrm{a}}$, Neil Karerakattil ${ }^{\mathrm{b}}$, Swarup Dey ${ }^{\mathrm{a}, \mathrm{c}}$, Devika Kishnan ${ }^{\mathrm{d}}$, and Rizal F. Hariadi ${ }^{(\mathbb{D} a, b, 1}$ \\ ${ }^{a} B i o d e s i g n$ Center for Molecular Design and Biomimetics (at the Biodesign Institute) at Arizona State University, Tempe, AZ \\ 85287, USA.; ' ${ }^{2}$ Department of Physics, Arizona State University, Tempe, AZ 85287, USA.; ' School of Molecular Sciences, \\ Arizona State University, Tempe, AZ 85287, USA.; ${ }^{\mathrm{d} S c h o o l}$ of Biological and Health System Engineering, Arizona State \\ University, Tempe, AZ 85287, USA.
}

${ }^{1}$ To whom correspondence should be addressed. E-mail: rhariadi@asu.edu

This manuscript was compiled on December 19, 2021

In vitro gliding assay is a well-established assay for determining the activity of protein motors, such as actin-associated myosins and microtubule-associated kinesins and dyneins. In one of the conventional methods, protein motors are immobilized onto a nitrocellulose-coated coverslip and it propels actin filaments in the presence of ATP. Gliding assays also serve as the foundation for protein-motor-based nanotechnological devices such as biosensing and sorting. However, the preparation of nitrocellulose-coated coverslips produces rough surfaces and in some protocols, requires up to an overnight incubation for the nitrocellulose film to completely dry. Furthermore, the nitrocellulose matrix exhibits high background autofluorescence, which reduces the signal-to-noise ratio of fluorescence images. Here, we investigated the use of hexamethyldisilazane (HMDS) to study actomyosin function and characterized its physical properties on glass coverslips and glass capillary tubes. We showed that the total preparation time to coat a coverslip with HMDS is $<30$ minutes and generates an atomically-flat surface with $\ll 0.1 \times$ of autofluorescence than HMDS. In addition, HMDS does not interfere with myosin function, which is indicated by the similar actin gliding speed when compared with nitrocellulose. Our results show that HMDS vapor deposition is a more favorable surface treatment to nitrocellulose for in vitro gliding assay and potentially for a broad class of biophysics experiments that require protein immobilization on hydrophobic surfaces.

\section{Introduction}

$\mathbf{M}$ olecular motors are involved in many cellular functions including mediating active cargo transport, muscle contraction, and cell division. ${ }^{1}$ Motor proteins such as myosins form a group and they walk on actin filaments to perform those processes. In vitro gliding assays are often used ${ }^{2-7}$ to elucidate the underlying mechanism and principles of the ATP-driven actin-myosin interaction. In this assay, isolated myosin or its fragments are adsorbed onto a treated glass coverslip where they propel the gliding of fluorescent-labeled actin filaments in the presence of ATP. ${ }^{8}$ In addition to providing considerable insight into the fundamentals of actomyosin biophysics, in vitro gliding assays have been proposed to serve as an active transport system in artificial environments for biomedical devices and molecular cybernetics. ${ }^{9-13}$

In conventional in vitro gliding assays, glass coverslips and slides are functionalized with nitrocellulose to bind myosin motors for in vitro gliding assay experiments. Nitrocellulose (Fig. 1A) is a polymer that is derived by nitrating cellulose via chemical a reaction involving a mixture of nitric acid and sulfuric acid. However, the preparation of coverslips with nitrocellulose is requires an overnight drying step and produces coated glass surfaces with a high background autofluorescence. Furthermore, gliding assays with nitrocellulose tend to give a lower fraction of motile filaments ${ }^{14-16}$ 


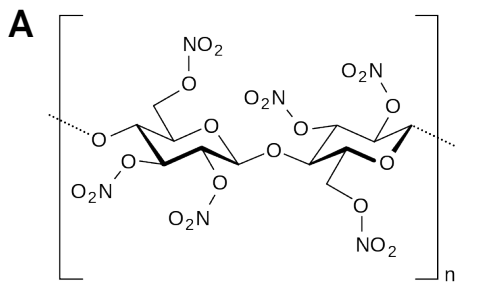

Nitrocellulose
B

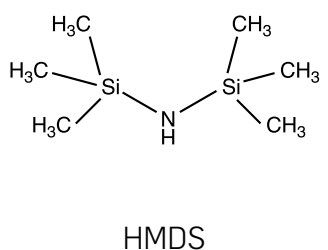

C

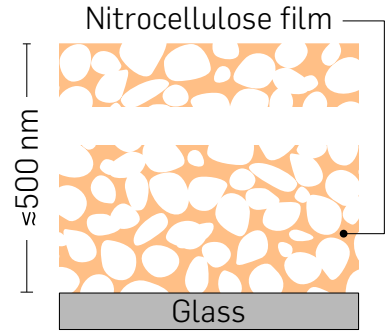

D

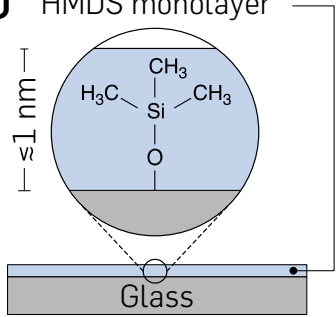

\section{E Coverslip preparation workflow}

\section{Nitrocellulose}

Day 1

Day 2

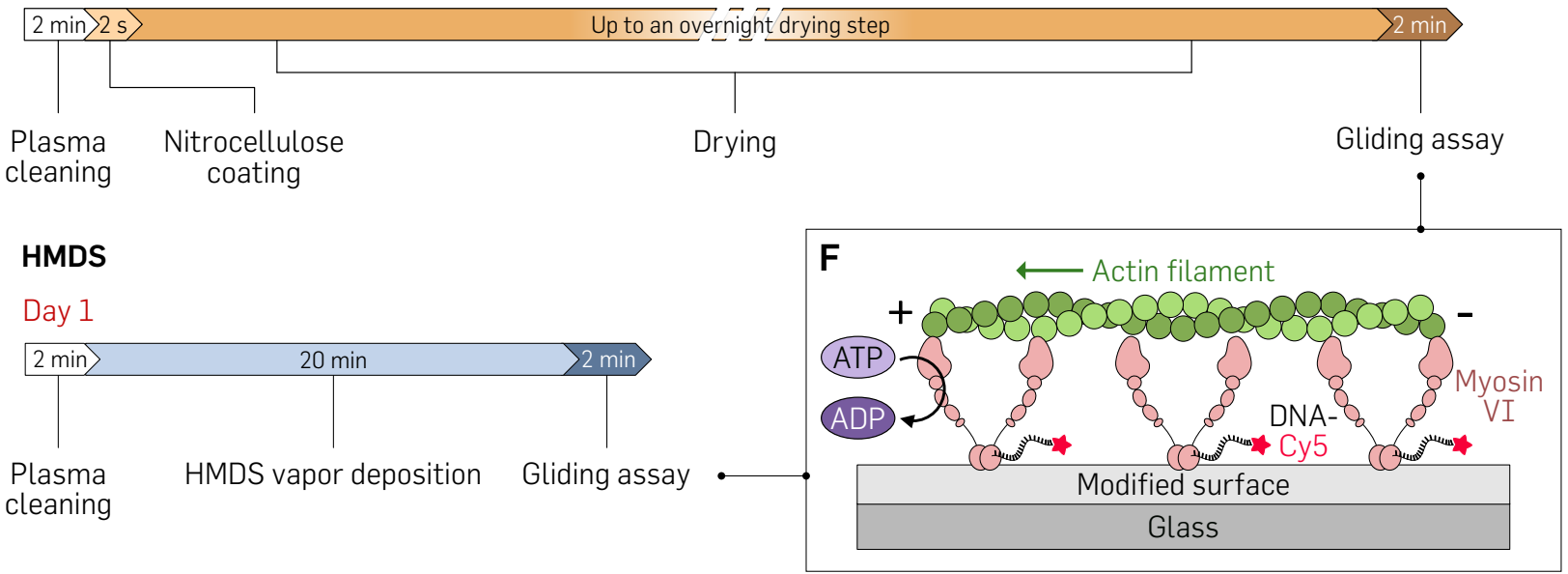

Fig. 1 Nitrocellulose and HMDS surface coatings for gliding assays. Chemical structures of nitrocellulose (A) and HMDS (B). Workflow schematics of coverslip modification with nitrocellulose (C) and HMDS (D). (E) Schematic of gliding assay with HMDS-coated coverslip. For schematic clarity, the relative orientation of myosin VI motors on HMDS was oversimplified. Mode of myosin VI absorbtion is unknown, but is expected to be stochastic.

Using silanes to coat coverslips has been shown to increase the gliding speed and gliding duration of actin filaments. ${ }^{6,14,16,17}$ Among these studies, it is shown that trimethylchlorosilane is superior in improving the fidelity of gliding assay and gives the most reproducible result compared to other silanes such as octadecylchlorosilane, dimethyldichlorosilane ${ }^{14}$, triethylchlorosilane, tripropylchlorosilane, tributylchlorosilane, phenyldimethylchlorosilane, and diphenylmethylchlorosilane ${ }^{16}$. The silanization process using these chemicals involves a lot of steps, including but not limited to piranha cleaning, then dipping the coverslips into silane/chlorofom mixtures.

Hexamethyldisilazane (HMDS; Fig. 1B) is one of the silane reagents that derivatizes surfaces through the formation of trimethylsilyl groups. ${ }^{18}$ In contrast to nitrocellulose that forms a disordered matrix ${ }^{19}$ (Fig. 1C), HMDS chemically bonds as a monolayer on the surfaces (Fig. 1D). HMDS reacts with oxidized surfaces, releasing ammonia, and forming a monolayer that consists of non-polar trimethylsilyl groups away from the substrate. This reaction forms a highly hydrophobic surface, which is a preferable condition to adsorb biomolecules with hydrophobic patches and to a lesser degree, to bind net-hydrophilic biomolecules. For an example, the bond between an HMDS-coated glass surface and $100 \mathrm{~nm} \times 100 \mathrm{~m}$, highly charged DNA origami nanostructures can be displaced by a series of wash step with Tween-20 detergent containing buffer. ${ }^{20,21}$ However, the use of HMDS for in vitro gliding assays of protein motors has not been demonstrated.

Derivatization of surfaces with HMDS can be easily done through chemical vapor deposition of a negatively-charged surface for less than $30 \mathrm{~min}$, which is significantly faster than nitrocellulose coating that 
requires up to an overnight drying before it is ready for functional assays (Fig. $1 \mathbf{E}$ and $\mathbf{F}$ ). Silanization with HMDS does not require glass coverslip to undergo an extensive cleaning procedure with piranha solution and plasma cleaning is sufficient for this modification. ${ }^{20} \mathrm{In}$ addition, the versatility of vapor deposition has the potential to modify less-exposed surfaces such as narrow capillary tubes that might be challenging to modify with viscous nitrocellulose.

Here, we evaluate the use of HMDS as a substrate to immobilize myosin by characterizing the physical properties such as hydrophobicity, surface height distribution, and autofluorescence of the modified coverslips. Then, we have compared the motility of actin on glass surfaces treated with HMDS and nitrocellulose. We tested our method to modify capillary tubes for gliding assays. We used myosin VI dimer constructs as a model system for motor proteins. We show that HMDS vapor deposition can modify exposed and less-exposed glass surfaces to allow immobilization of motor proteins. The immobilized myosins were functional for in vitro gliding assays.

\section{Results}

HMDS vapor deposition forms a uniform layer on glass coverslips. We deposited monolayer HMDS on Corning D263 glass coverslips using the vapor deposition method at room temperature and atmospheric pressure (Materials and Methods; Fig. 1). We also deposited nitrocellulose on the same model of coverslips. The overall process of surface modification with HMDS takes less than $30 \mathrm{~min}$, while the nitrocellulose deposition process takes 16 hours until the coverslip is ready to use (Fig. 1E). Representative AFM images of both surface coating techniques are presented in Fig. $2 \mathbf{A}$ and B with the same color scale to allow a direct comparison of the samples. We increased the range of the color scale to reduce the appearance of noise for a better visual analysis. AFM images with real-time scaling mode show a periodic noise (Fig. S1 and S2). HMDS-coated surfaces are cleaner than nitrocellulose-coated surfaces as indicated by the presence of blobs (bright spots) on nitrocellulose-coated surfaces (Fig. 2B). There is a clear difference of the surface height distribution between HMDS-coated surface (Fig. 2C) and nitrocellulose-coated surfaces (Fig. 2D). There is an asymmetry observed in the histogram of nitrocellulose-coated surface heights and they are more variable compared to the heights of HMDS-coated surfaces.

TIRF assays of partially modified glass coverslips with nitrocellulose show that the mean height of the deposited nitrocellulose is $456 \pm 125 \mathrm{~nm}$ thick, which indicates that it has multilayer thickness. In contrast, AFM characterization of partially modified glass coverslips (data not shown) shows that the deposited HMDS layer is 1 monolayer thick $(<1 \mathrm{~nm})$, which is three orders of magnitude thinner than nitrocellulose films (Fig. 2E). The monolayer formation of HMDS is consistent with previous reports that HMDS interacts with activated $\mathrm{SiO}_{2}$ and not with trimethylsilyl groups.

HMDS modifies the hydrophilic plasma-cleaned surface to a hydrophobic surface. Plasma-cleaning (PC) reduces the contact angle of untreated (non-plasma-cleaned; NPC) glass surfaces from $40.4^{\circ} \pm 1.9^{\circ}(\mathrm{N}=$ 3 ) into completely hydrophilic $\left(0^{\circ}\right.$ contact angle; $\mathrm{N}=3$; Fig. $3 \mathbf{F}$ and $\left.\mathbf{G}\right)$. After vapor deposition of HMDS, PC glass surface becomes hydrophobic which is indicated by the increase of contact angle to $64.1^{\circ} \pm 3.2^{\circ}(\mathrm{N}=3)$. Surprisingly, HMDS vapor deposition of inactive NPC glass also renders the surface hydrophobic $\left(70.7^{\circ} \pm 1.8^{\circ}(\mathrm{N}=3\right.$; Fig. $2 \mathbf{H})$, which is significantly higher $(p=0.037)$ than HMDS-coated PC glass (Fig. 2I). The similar contact angle of nitrocellulose-coated NPC glass $\left(59.5^{\circ} \pm 0.1^{\circ}\right)$ and PC glass $\left(61.1^{\circ} \pm 2.0^{\circ}\right)$ shows that plasma cleaning of glass does not affect the hydrophobicity of nitrocellulose coating $(p=0.236$; Fig. 2J-L). The contact angles of HMDS-coated PC surfaces are on par with the contact angle of both nitrocellulose-coated NPC and PC surfaces.

HMDS have lower autofluorescence than nitrocellulose. We quantified the autofluorescence intensity and photo-bleaching of nitrocellulose- and HMDS-coated coverslips in a 2-min period (Movies S1-S3). Nitrocellulose-coated surfaces exhibit higher background intensity than HMDS-coated surfaces and bare glass (Fig. 2M). In addition to that, HMDS-coated surfaces have similar intensities as the standard bare 


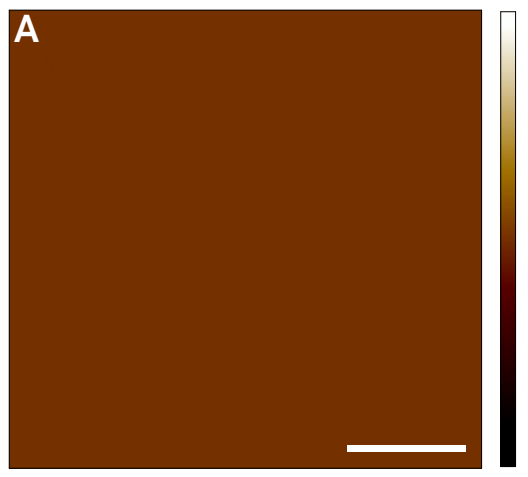

C
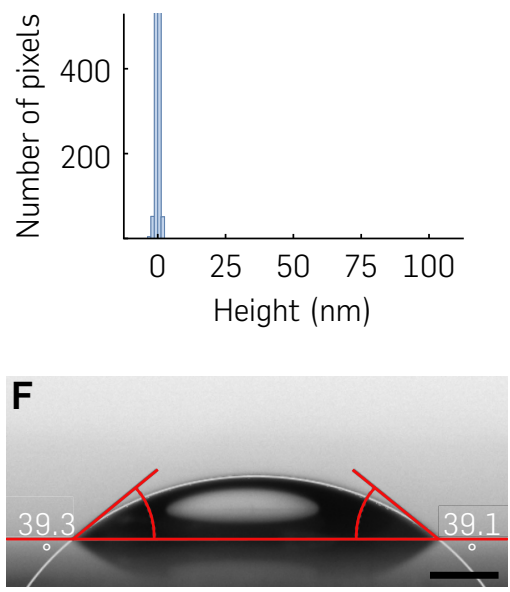

NPC glass

G

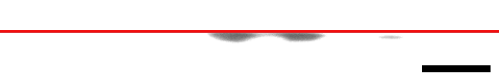

PC glass
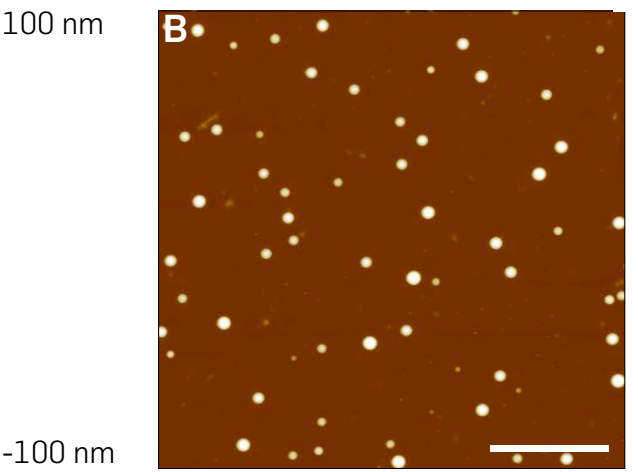

D

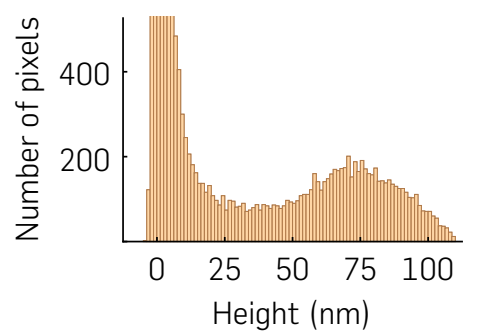

$100 \mathrm{~nm}$

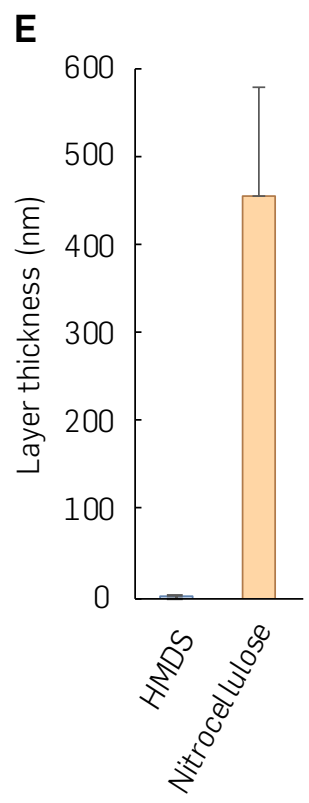

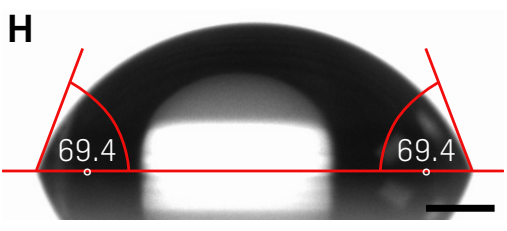

NPC glass + HMDS

I

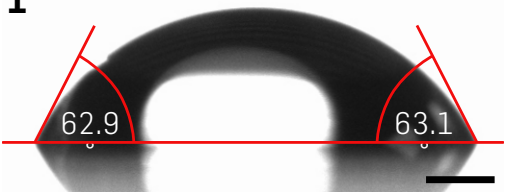

PC glass + HMDS

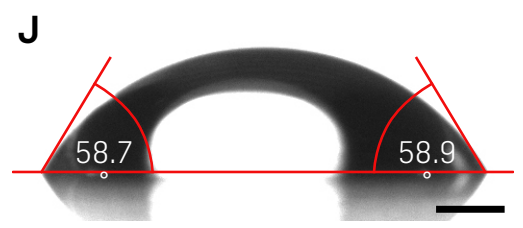

NPC glass + Nitrocellulose

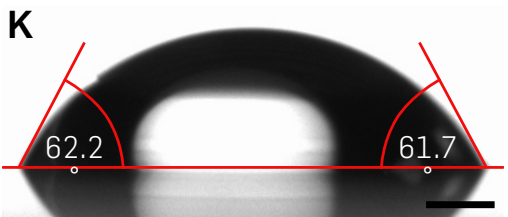

PC glass + Nitrocellulose
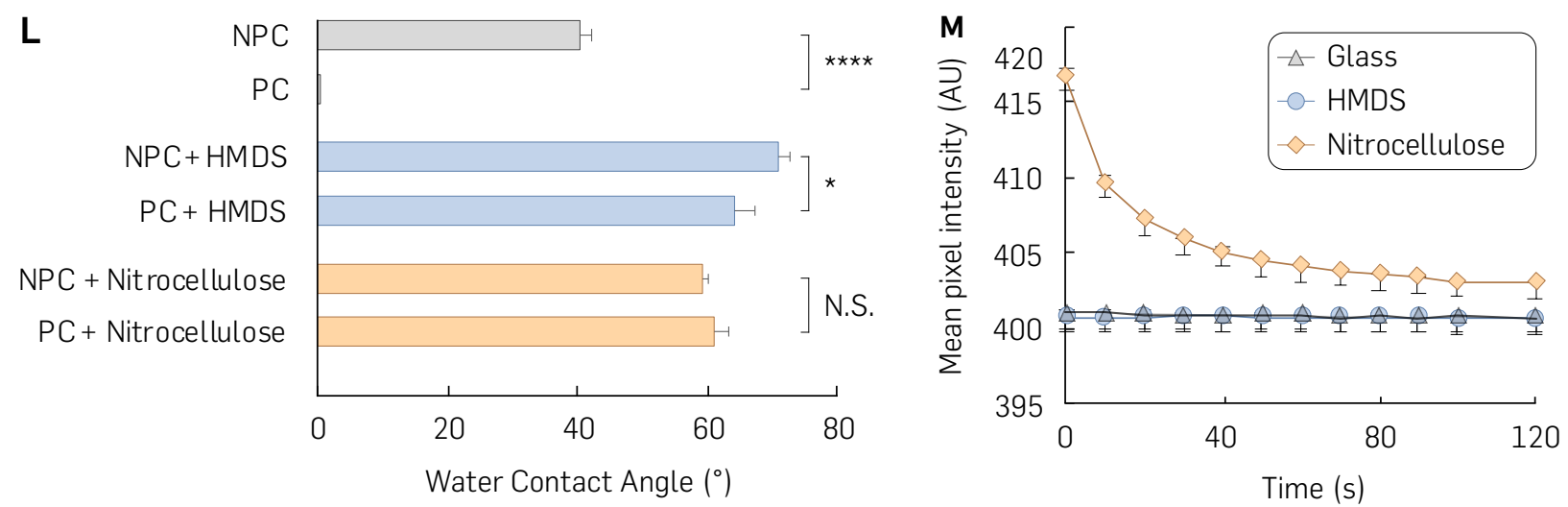

Fig. 2 Physical characterization of HMDS-coated and nitrocellulose-coated glass coverslips. (A-B) Representative AFM images of HMDS-coated (A) and nitrocellulose-coated (B) surfaces. Scale bar represents $5 \mu \mathrm{m}$. (C-D) Height distribution of HMDS-coated (C) and nitrocellulose-coated (D) surfaces. (E) Film thickness of treated surfaces $(\mathrm{N}=3)$. $(\mathbf{F}-\mathbf{K})$ Representative images of water droplets on non-plasma-cleaned $(\mathrm{NPC} ; \mathbf{F})$, plasma-cleaned (NPC; $\mathbf{G})$, NPC glass treated with HMDS $(\mathbf{H})$, PC glass treated with HMDS $(\mathbf{I})$, NPC glass coated with nitrocellulose $(\mathbf{J})$, and PC glass coated with nitrocellulose $(\mathbf{K})$. (L) Contact angle measurements of different treatments of glass coverslip $(\mathrm{N}=3$ samples). Scale bar represents $1 \mathrm{~mm}$. (M) Mean fluoroscence/pixel intensity of glass, HMDS-coated, and nitrocellulose-coated surfaces ( $\mathrm{N}=3$ field of views). Error bars are \pm SD. 
glass. The intensity of nitrocellulose-coated surfaces is reduced over time. However, even after 2 min, the intensity of nitrocellulose background is still higher than the glass surface.

Myosin-actin gliding assay is compatible with HMDS. Fluorescent time-lapse images of gliding assays in the absence of ATP revealed that myosin VI motors bind to HMDS (Fig. 3A). This is indicated by the binding of actin filaments to HMDS surfaces deposited with myosin VI motors. However, at $0 \mathrm{mM}$ ATP actin filaments do not glide along the myosin surface $(0 \mu \mathrm{ms}$ gliding speed $)$. As seen in Fig. 3B, actin filaments are not adsorbed onto HMDS-coated surfaces in the absence of myosin motors. This result is similar to when common passivating agent bovine serum albumin (BSA) was used to coat the glass surfaces and HMDS-coated surfaces (Fig. 3C and D). As expected, actin filaments are adsorbed to bare plasma-cleaned glass surfaces (Fig. 3E). This result suggests that HMDS is a compatible substrate to use for the in vitro gliding assay because it does not interact with actin filaments on its own.

Gliding assay (Fig. 2F) shows that at $2 \mathrm{mM}$ ATP, there is gliding of actin filaments which indicates that $\mathrm{t}$ he myosin are immobilized and functional when immobilized on HMDS- and nitrocellulose-coated surfaces (Fig. 2G, H, Movies S4 and S5). The fraction of motile filaments on HMDS-coated surfaces does not have any significant difference $(p$ value $=0.15)$ with that on nitrocellulose-coated surfaces $(\mathbf{F i g} .3 \mathbf{I})$. Accordingly, the average gliding speed on HMDS-coated surfaces is similar $(63 \pm 14 \mathrm{~nm} / \mathrm{s})$ to that on nitrocellulose-coated surfaces $(64 \pm 14 \mathrm{~nm} / \mathrm{s})$. In addition, the actin-length-dependent gliding velocities of myosin VI on HMDS-and nitrocellulose-coated surfaces are qualitatively similar (Fig. 3J).

HMDS functionalization is compatible with commonly used passivating agents such as BSA and Tween-20 (Table 1). Here, the coverslips were plasma-cleaned before any treatment. HMDS-coated surfaces were pretreated with passivating agents before myosin VI deposition. After myosin deposition on the treated surfaces, we measured the actin gliding speed. Pre-treatment with BSA does not outcompete myosin VI binding to HMDS, indicated by the statistically identical gliding speed between experiments using BSA (139 $\pm 25 \mathrm{~nm} / \mathrm{s}(\mathrm{N}=50$ filaments); Movie S6). The same set of experiments without BSA lead to slower mean gliding speed $(63 \pm 14 \mathrm{~nm} / \mathrm{s}(\mathrm{N}=50$ filaments); Movie $\mathrm{S} 4)$ presumably due to the interactions between HMDS surface, myosin VI, and the gliding actin filaments. Control experiments with BSA-coated surface and in the absence of HMDS coating (Movie S7) show very brief ( $<1$ sec) gliding actin filaments that is consistent with the absence of bound myosin VI on BSA-passivated coverslips. Tween-20 inhibits myosin VI binding to HMDS-coated surfaces and plasma cleaned glass as there were no actin binding and gliding observed on both surfaces (Movies S8 and S9). Within our experiments, the observed actin gliding speed is the slowest when the glass surface was only plasma-cleaned without further surface treatment ( $40 \pm 12 \mathrm{~nm} / \mathrm{s} ; \mathrm{N}=20$ filaments; Movie S10), presumably due to the strong interactions between the unpassivated plasma-cleaned surface, myosin VI, and actin filaments.

\begin{tabular}{cccc|rr}
\hline \multicolumn{4}{c}{ Sample preparation } & \multicolumn{2}{c}{ Measurement } \\
\hline Step 1 & Step 2 & Step 3 & Step 4 & & \\
Plasma-cleaning & HMDS & Passivating agent & myosin VI & Gliding speed (nm/s) & Movie \\
\hline \hline+ & + & - & + & $63 \pm 14$ & S4 \\
+ & + & BSA & + & $139 \pm 25$ & S6 \\
+ & + & Tween-20 & + & Not observed & S8 \\
+ & - & BSA & + & Not observed & S7 \\
+ & - & Tween-20 & + & Not observed & S9 \\
+ & - & - & + & $40 \pm 12$ & S10 \\
\hline
\end{tabular}

Table 1 Gliding speed of indicated experiment using different surface passivating strategies ( $\mathbf{N} \geq \mathbf{2 0}$ filaments). The addition of Tween-20 detergent after HMDS vapor deposition block myosin VI from binding to the passivated surfaces. 
Surface modification:

HMDS

HMDS

BSA

HMDS + BSA

Myosin VI
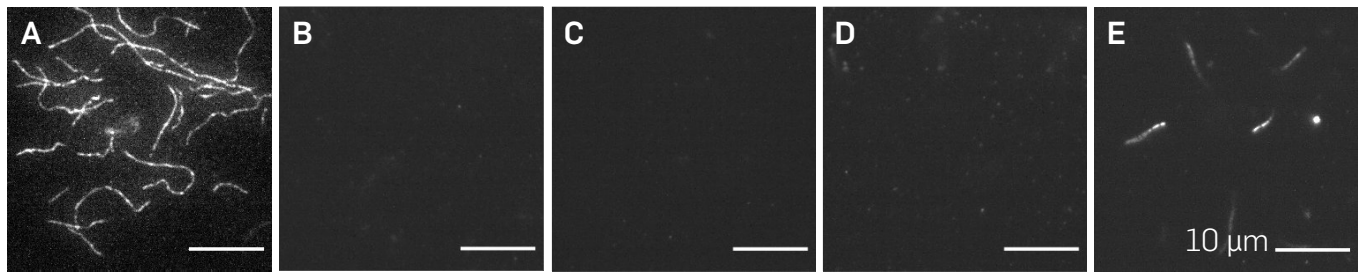

\section{F Gliding assay protocol}
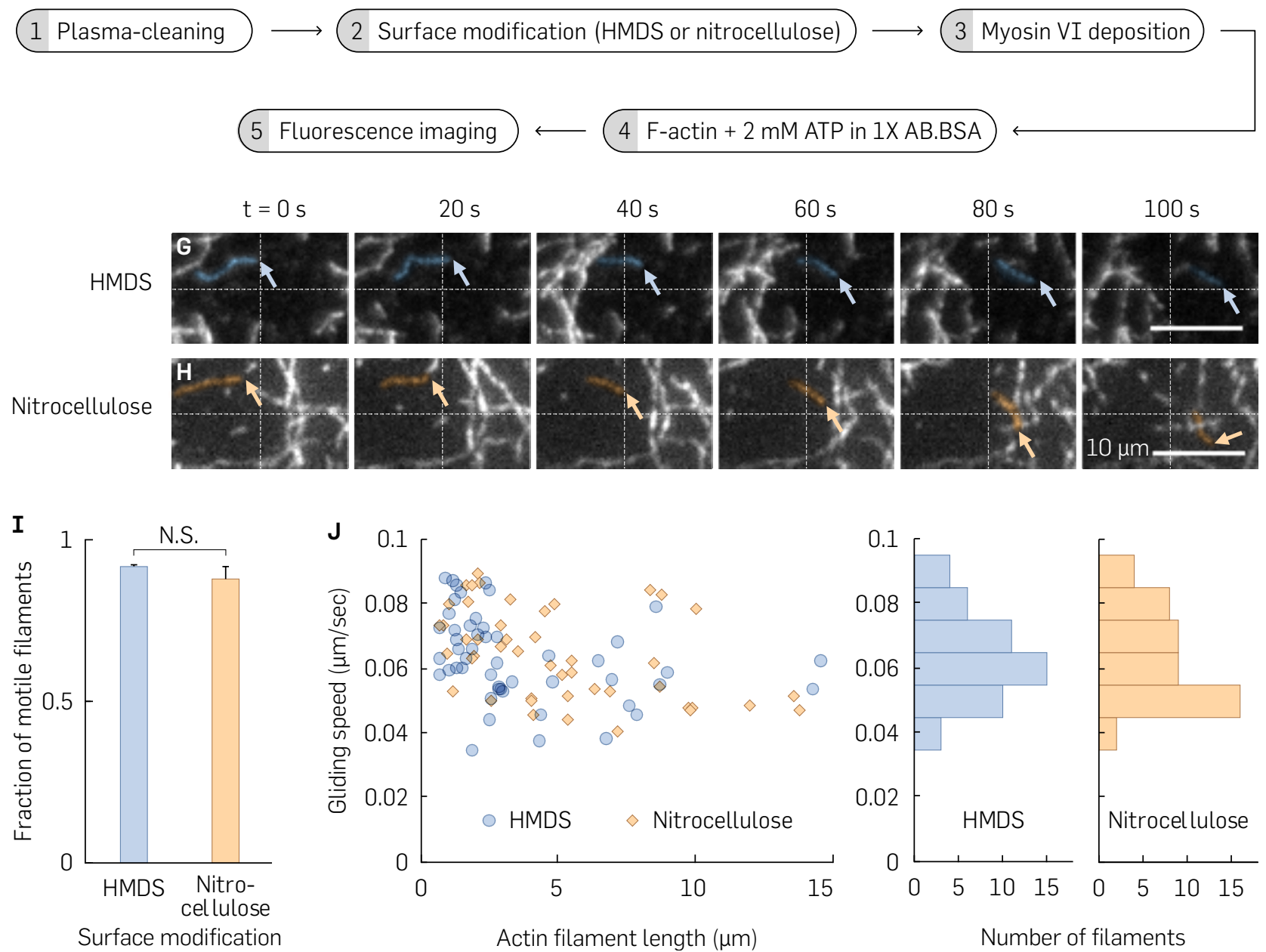

Fig. 3 Actomyosin function on different coverslip surface modifications. (A) Fluorescence image of rhodamine-labelled F-actin on HMDS-coated myosin VI surface with $0 \mathrm{mM}$ ATP. Fluorescence images of rhodamine-labelled F-actin adsorption on HMDS- (B), BSA- (C), HMDS + BSA-coated (D), and plasma-cleaned glass (E) surfaces without myosin VI at $2 \mathrm{mM}$ ATP. (F) Gliding assay protocol to measure actin gliding speeds and fraction of motile filaments. Fluorescence micrograph time series at $20 \mathrm{~s}$ intervals displaying motile and non-motile actin filaments on HMDS-coated $(\mathbf{G})$ and nitrocellulose-coated $(\mathbf{H})$ surfaces (Movies S4-S5). Scale bar represents $5 \mu \mathrm{m}$. (I) Fraction of motile filaments (motile filaments divided by total number of filaments on a $20 \mu \mathrm{m} \times 20 \mu \mathrm{m}$ area, $\mathrm{N}=3$ fields of views) on HMDS-coated and nitrocellulose-coated surfaces. (J) Gliding speed of actin filaments on HMDS surface (blue) and nitrocellulose (orange). Error bars are $\pm \mathrm{SD}(\mathrm{N}=50$ filaments $)$.

HMDS vapor deposition modifies the surface hydrophobicity of capillary tubes. Vapor deposition of HMDS can modify geometrically less exposed surfaces, namely, the inner surface capillary tubes. Here, 
instead of using capillary tubes with circular cross sections, we evaluated the HMDS surface chemistry of the inner surface of capillary tubes with a uniform rectangular cross section. The rectangular cross section enables microscopy assays for measuring the speed of gliding actin filaments. The inner dimensions of the capillary tubes are $2.0 \mathrm{~mm} \times 0.20 \mathrm{~mm} \times 50 \mathrm{~mm}$. Fig. $4 \mathbf{A}$ compares HMDS and nitrocellulose modifications of rectangular capillary tubes. The height of the dyed water was recorded by measuring the distance from the bottom of the capillary tube to the meniscus of the dyed water. The more hydrophilic the interior walls of the capillary tube are, the higher the water rises in the capillary tubes: Plasma cleaning of the untreated capillary surface increased the capillary rise to the other end of the tube (Fig. 4A). Fig. 2 shows that hydrophobicity manifests in a larger contact angle. The plasma cleaned capillary tubes were treated with HMDS or nitrocellulose and both of these treatments made the surfaces hydrophobic (Fig. 4A and B). In the context of vertical capillary tubes (Fig. 4), Jurin's Law predicts the height $h$ of the water given the contact angle $\theta$, surface tension $\gamma$, acceleration due to gravity $g$, density $\rho$, and tube radius $r$. More importantly, it establishes a relation between height and contact angle that is used to convert the height data to contact angles.

$$
h=\frac{2 \gamma \cos \theta}{\rho g r}
$$

The caveat of our model is that Jurin's law applies for cylinders, which is different than the rectangular cross section of our capillary tubes. Consequently, the rectangular tubes used in this experiment do not have a radius $r$ that can be found directly. We used 2 physical models which provide upper and lower bound estimates. The first assumption is a cylindrical cross section and equates the areas such that width $\times$ length $=\pi r^{2}$. Using the first model, the effective $r$ is estimated to be $0.357 \mathrm{~mm}$. The second assumption is where we model the capillary tube as two parallel plates spaced closely together. The capillary rise for two parallel plates is given by a similar formula where $r$ is replaced with the spacing between the two plates $(d=0.2 \mathrm{~mm})$. Note that Jurin's law only works for heights that produce values within the range accepted by the cosine inverse function. In addition, the water height is limited by the height of the capillary tube, so calculations with heights that exceed the max height (i.e. all plasma-cleaned tubes) have been excluded from the data (undefined; Fig. 4B).

The final contact angles of both glass coverslips and capillary tubes after HMDS treatment are similar $\left(53.0^{\circ}-69.9^{\circ}\right)$. This indicates that our HMDS vapor deposition is able to modify unexposed glass or unconventional 3D chamber. Nitrocellulose-coated capillary tubes have a slightly higher contact angle $\left(72.2^{\circ}-79.9^{\circ}\right)$ than HMDS-coated capillary tubes. However, nitrocellulose coating had to be done with extra care to avoid clogging of the tubes.

HMDS surface modification is compatible with preparing the internal surface of rectangular capillary tubes for filament gliding assays with myosin VI. Fluorescent time-lapse images of gliding assay with HMDS-coated capillary tubes show that actin filaments glide along the surface (Fig. 4C, Movie S11). The gliding speed of the actin filaments on the capillary tube setup was measured to be $107 \pm 35 \mathrm{~nm} / \mathrm{sec}$ ( $N=29$ filaments), which is within $23 \%$ of the gliding speed observed from the HMDS-coated coverslip setup (139 $\pm 25 \mathrm{~nm} / \mathrm{sec}$; Table $\mathbf{1}$ (second row)). We observed a lack of actin filaments gliding in unmodified or plasma-cleaned tubes (Movies S12 and S13), similar to the low numbers of gliding in filaments in plasma-cleaned only coverslips (Table 1). These results demonstrated that the myosin VI binds to HMDS-treated rectangular capillary tubes as well. We were unable to get gliding assay data on nitrocellulose-coated capillary tubes because the nitrocellulose and gliding assay mix (Go mix; Supporting Information) was not able to flow through the capillary tubes.

\section{Discussion}

Here, we demonstrated a simple approach using HMDS vapor deposition to immobilize myosin motors. Our results show that HMDS can provide an atomically flat surface with lower autofluorescence and a faster 


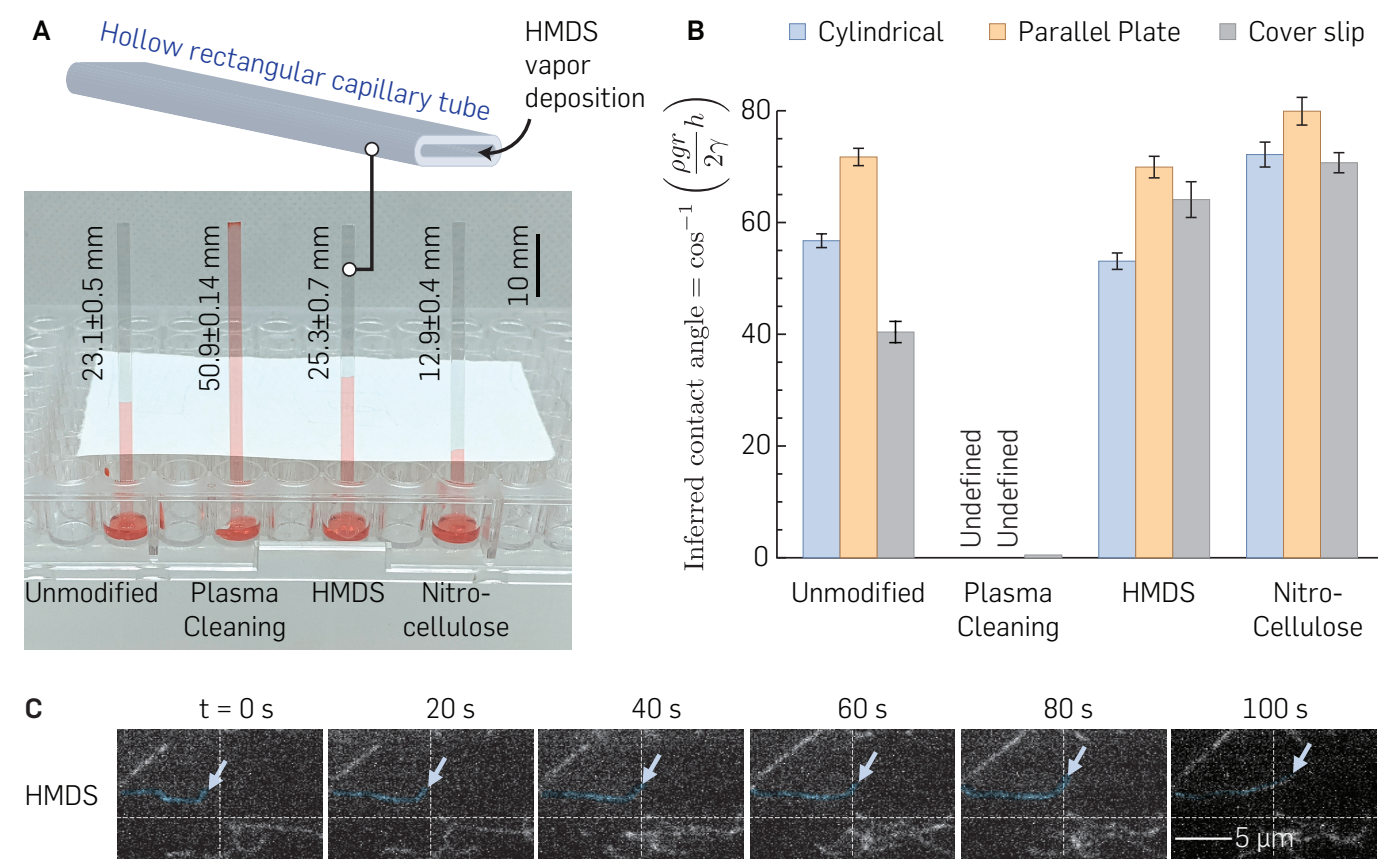

Fig. 4 Physical properties of modified capillary tubes and actomyosin function on HMDS-treated capillary tubes. (A) Capillary rise recorded for each step of the HMDS and nitrocellulose preparation $(\mathrm{N}=3)$. The image was adjusted to remove perspective distortion for accurate height comparison. (B) Inferred contact angles calculated from the capillary rise data. Results from cylindrical and parallel plate assumptions are compared to values obtained for coverslips. (C) Fluorescence micrograph time series of a gliding actin filament (highlighted in blue) relative to the non-motile actin filaments (Movies S11). Scale bar is $5 \mu \mathrm{m}$. Error bars are \pm SEM.

preparation time that is suitable for in vitro gliding assay. Compared to nitrocellulose-coated surfaces, HMDS-coated surfaces have improved physical properties for in vitro gliding assays. According to previous studies ${ }^{14}$, silanized surfaces are smoother than nitrocellulose-coated surfaces. The height of the nitrocellulose film was significantly thicker and more variable than the HMDS monolayer. One explanation is that, unlike nitrocellulose, HMDS cannot form a polymer on the negatively charged coverslip. Furthermore, in line with the idea that HMDS is the superior option for coverslip-coating, HMDS-coated surfaces have a significantly lower autofluorescence, which solves the problem of interference of background autofluorescence that persists in nitrocellulose coatings. HMDS-coated surfaces have similar hydrophobicity to nitrocellulose, which is appropriate for binding functional myosin VI molecules. Our silane coating with HMDS has a lower contact angle than that of TMCS $\left(76.4 \pm 1.6^{\circ}\right)$ reported in the earlier study. ${ }^{14}$ However, in comparison to the results in that study, our control nitrocellulose-coated surfaces also have a lower contact angle. This lower contact angle can possibly be attributed to the different type of coverslip and the treatment methods we used in our study.

We performed gliding assays on HMDS-coated surfaces to evaluate the compatibility of HMDS with actin filament - myosin VI interactions. The measured gliding speeds of actin filaments on myosin VI immobilized on HMDS-coated surfaces are within $98 \%$ with the gliding speeds on the standard nitrocellulose-coated surfaces (Fig. 3). These results are 21\% slower than the previously reported gliding speed measured using myosin VI-labeled DNA patterned on DNA nanotubes ${ }^{22}$ and two-dimensional DNA origami scaffolds ${ }^{23,24}$. We found that the average actin gliding speed increased when HMDS was preincubated with BSA before myosin VI immobilization. Similar result was observed in a previous study where passivation with alpha and whole casein increased the gliding speed of microtubules. ${ }^{25}$ This indicated that BSA has a marked effect on gliding speeds, which might be attributed to the lower density or number of myosin VI immobilized on the surface. With a large number of myosins, the actin gliding speeds is effectively independent of the 
number of proteins ${ }^{22}$. These results are $57 \%$ slower than the speeds of single-myosin VI movement on actin filaments. ${ }^{26}$ The speed decrease is similar to that previously reported for one to six myosin VI motors on two-dimensional actin networks. ${ }^{27}$ The selective adsorption of protein to HMDS can be explained by the different hydrophobicity and charge of myosin VI and actin filaments. We do not know the mode of myosin VI adsorption to the silanized surface. However, because the silanized surface is hydrophobic, the hydrophobic part of myosin VI (the tail domain ${ }^{28}$ ) is possibly bound to it through hydrophobic-hydrophobic interactions. Some studies suggest that hydrophobicity is among the underlying mechanisms of myosin molecules adsorption. ${ }^{29,30}$

In conclusion, HMDS can substitute nitrocellulose for in vitro gliding assay but with faster preparation time, no autofluoresence, and a atomically-flat surface without being restricted by the geometry of the sample chambers. Our tests with capillary tubes indicate that HMDS is a versatile surface modification that can be used for treating glass surfaces with unique geometries. Contact angles and successful gliding attempts confirm that plasma-cleaned glass is compatible with HMDS, even in tight chambers where flow is restricted. Since we use vapor deposition in a vacuum chamber, no flow is required for HMDS modification. The contact angle measurements that match the values obtained with coverslips and the observation of gliding confirms the consistency of this method. However, future research should investigate the mechanism of myosin VI binding to the silanized surface. It would be fruitful to explore the application of HMDS as a substrate for other motor proteins, such as kinesin and dynein.

\section{Acknowledgements}

The authors thank Sivaraj Sivaramakrishnan and Michael Ritt for providing the plasmid for myosin VI and Patrick Gleason for expressing calmodulin. We thank Carter Swanson, Biruni Hariadi, Daisuke Inoue, and Ruth Sommese for helpful discussions. AFM images were collected in the lab of Hao Yan. The proteins were expressed in a cell culture room facility provided by Center for Personalized Diagnostics in the Biodesign Institute at Arizona State University.

\section{Funding}

The work in Hariadi lab was supported by National Institutes of Health Director's New Innovator Award (1DP2AI144247) to RFH, Arizona Biomedical Research Consortium (ADHS17-00007401), and National Science Foundation (2027215) to RFH.

\section{Author contributions}

I.F.T. and R.F.H. contributed to the experimental design, planning, and execution. I.F.T. and N.K. performed the experiments involving capillary tubes. D.K. collected AFM images. S.D. measured the contact angle of modified glass surfaces. I.F.T., N.K., and R.F.H. wrote the manuscript and prepared figures.

\section{Conflict of interest}

The author declares there is no conflict of interest.

Data and material availability. The images, raw data files, and the Mathematica code for performing the data analysis are on a GitHub repository.

https://github.com/bionicslab/HMDS-manuscript

\section{References}

1. M. Amanda Hartman and James A. Spudich. The myosin superfamily at a glance. J Cell Sci, 125(7):1627-1632, 2012 . 
2. Earl Homsher, Bibiana Kim, Alyona Bobkova, and Larry S. Tobacman. Calcium regulation of thin filament movement in an in vitro motility assay. Biophys J, 70(4):1881-1892, 1996.

3. Ida Lister, Stephan Schmitz, Matthew Walker, John Trinick, Folma Buss, Claudia Veigel, and John Kendrick-Jones. A monomeric myosin VI with a large working stroke. EMBO J, 23(8):1729-1738, 42004.

4. Taro Q.P. Uyeda, Paul D. Abramson, and James A. Spudich. The neck region of the myosin motor domain acts as a lever arm to generate movement. Proc Natl Acad Sci USA, 93(9):4459-4464, 41996.

5. J. E. Molloy, J. E. Burns, B. Kendrick-Jones, R. T. Tregear, and D. C.S. White. Movement and force produced by a single myosin head. Nature, 378(6553):209-212, 111995.

6. Yoshie Harada, Katsuhiko Sakurada, Toshiaki Aoki, David D. Thomas, and Toshio Yanagida. Mechanochemical coupling in actomyosin energy transduction studied by in vitro movement assay. J Mol Biol, 216(1):49-68, 11 1990.

7. Coleen T. Murphy, Ronald S. Rock, and James A. Spudich. A myosin II mutation uncouples ATPase activity from motility and shortens step size. Nat Cell Biol, 3(3):311-315, 2001.

8. Stephen J. Kron, Yoko Y. Toyoshima, Taro Q.P. Uyeda, and James A. Spudich. Assays for actin sliding movement over myosin-coated surfaces. Method Enzymol, 196(C):399-416, 11991.

9. Dan V. Nicolau, Mercy Lard, Till Korten, Falco C.M.J.M. Van Delft, Malin Persson, Elina Bengtsson, Alf Månsson, Stefan Diez, Heiner Linke, and Dan V. Nicolau. Parallel computation with molecular-motor-propelled agents in nanofabricated networks. Proc Natl Acad Sci USA, 113(10):2591-2596, 32016.

10. Chih Ting Lin, Ming Tse Kao, Katsuo Kurabayashi, and Edgar Meyhofer. Self-contained, biomolecular motor-driven protein sorting and concentrating in an ultrasensitive microfluidic chip. Nano Lett, 8(4):1041-1046, 42008.

11. Thorsten Fischer, Ashutosh Agarwal, and Henry Hess. A smart dust biosensor powered by kinesin motors. Nat Nanotechnol, 4(3):162-166, 12009.

12. Saroj Kumar, Gloria Milani, Hideyo Takatsuki, Tobia Lana, Malin Persson, Chiara Frasson, Geertruy Te Kronnie, and Alf Månsson. Sensing protein antigen and microvesicle analytes using high-capacity biopolymer nano-carriers. Analyst, 141(3):836-846, 22016.

13. Martin G. L. Van den Heuvel and Cees Dekker. Motor proteins at work for nanotechnology. Science, 317(5836):333-336, 2007.

14. Mark Sundberg, Jenny P. Rosengren, Richard Bunk, Joakim Lindahl, Ian A. Nicholls, Sven Tågerud, Pär Omling, Lars Montelius, and Alf Månsson. Silanized surfaces for in vitro studies of actomyosin function and nanotechnology applications. Anal Biochem, 323(1):127-138, 122003.

15. Mohammad A. Rahman, Aseem Salhotra, and Alf Månsson. Comparative analysis of widely used methods to remove nonfunctional myosin heads for the in vitro motility assay. J Muscle Res Cell M, 39(5-6):175-187, 12 2018.

16. Nuria Albet-Torres, John O’Mahony, Christy Charlton, Martina Balaz, Patricia Lisboa, Teodor Aastrup, Alf Månsson, and Ian A. Nicholls. Mode of heavy meromyosin adsorption and motor function correlated with surface hydrophobicity and charge. Langmuir, 23(22):11147-11156, 102007.

17. Hans M. Warrick, Robert M. Simmons, Jeffrey T. Finer, Taro Q.P. Uyeda, Steven Chu, and James A. Spudich. Chapter 1 In Vitro Methods for Measuring Force and Velocity of the Actin-Myosin Interaction Using Purified Proteins. 1993.

18. Benjamin A. Anderson and Vikas Sikervar. Hexamethyldisilazane. pages 1-4.

19. Michelle L. Tonkin, Magali Roques, Mauld H. Lamarque, Martine Pugnière, Dominique Douguet, Joanna Crawford, Maryse Lebrun, and Martin J. Boulanger. Host cell invasion by apicomplexan parasites: Insights from the co-structure of AMA1 with a RON2 peptide. Science, 333(6041):463-467, 72011.

20. Ashwin Gopinath and Paul WK Rothemund. Optimized assembly and covalent coupling of single-molecule DNA origami nanoarrays. ACS Nano, 8(12):12030-12040, 2014.

21. Rishabh M. Shetty, Sarah R. Brady, Paul W. K. Rothemund, Rizal F. Hariadi, and Ashwin Gopinath. Low-cost, bottom-up fabrication of large-scale single-molecule nanoarrays by DNA origami placement. bioRxiv, 2020.

22. R. F. Hariadi, R. F. Sommese, A. S. Adhikari, R. E. Taylor, S. Sutton, J. A. Spudich, and S. Sivaramakrishnan. Mechanical coordination in motor ensembles revealed using engineered artificial myosin filaments. Nat Nanotechnol, 10(8):696-700, 2015.

23. Rizal F Hariadi, Mario Cale, and Sivaraj Sivaramakrishnan. Myosin lever arm directs collective motion on cellular actin network. Proc Natl Acad Sci USA, 111(11):4091-4096, 2014.

24. Rizal F Hariadi, Ruth F Sommese, and Sivaraj Sivaramakrishnan. Tuning myosin-driven sorting on cellular actin networks. eLife, 4:e05472, 2015.

25. Andy Maloney, Lawrence J Herskowitz, and Steven J Koch. Effects of surface passivation on gliding motility 
assays. PLoS One, 6(6):e19522, 2011.

26. M. Yusuf Ali, Guy G. Kennedy, Daniel Safer, Kathleen M. Trybus, H. Lee Sweeney, and David M. Warshaw. Myosin Va and myosin VI coordinate their steps while engaged in an in vitro tug of war during cargo transport. Proc Natl Acad Sci USA, 108(34):E535-E541, 82011.

27. Rizal F. Hariadi, Mario Cale, and Sivaraj Sivaramakrishnan. Myosin lever arm directs collective motion on cellular actin network. Proc Natl Acad Sci USA, 111(11):4091-4096, 2014.

28. Hyeong Jun Kim, Jen Hsin, Yanxin Liu, Paul R. Selvin, and Klaus Schulten. Formation of salt bridges mediates internal dimerization of myosin VI medial tail domain. Structure, 18(11):1443-1449, 112010.

29. Kristi L. Hanson, Florin Fulga, Serban Dobroiu, Gerardin Solana, Ondrej Kaspar, Viola Tokarova, and Dan V. Nicolau. Polymer surface properties control the function of heavy meromyosin in dynamic nanodevices. Biosens Bioelectron, 93:305-314, 72017.

30. Kazuhiro Nakanishi, Takaharu Sakiyama, and Koreyoshi Imamura. On the adsorption of proteins on solid surfaces, a common but very complicated phenomenon. J Biosci Bioeng, 91(3):233-244, 2001.

31. Johannes Schindelin, Ignacio Arganda-Carreras, Erwin Frise, Verena Kaynig, Mark Longair, Tobias Pietzsch, Stephan Preibisch, Curtis Rueden, Stephan Saalfeld, Benjamin Schmid, Jean Yves Tinevez, Daniel James White, Volker Hartenstein, Kevin Eliceiri, Pavel Tomancak, and Albert Cardona. Fiji: An open-source platform for biological-image analysis. Nat Methods, 9(7):676-682, 72012.

32. Erik Meijering, Oleh Dzyubachyk, and Ihor Smal. Methods for cell and particle tracking. In Method Enzymol, volume 504, pages 183-200. Academic Press Inc., 2012.

\section{Materials and Methods}

Buffers. $1 \times$ assay buffer (AB): $25 \mathrm{mM}$ imidazole (pH 7.5), $4 \mathrm{mM}$ Magnesium Chloride, $1 \mathrm{mM}$ Ethylene glycol-bis( $\beta$-aminoethyl ether)-N,N,N',N'-tetraacetic acid tetrasodium salt, $25 \mathrm{mM}$ Potassium Chloride, $10 \mathrm{mM}$ Dithiothreitol. $1 \times$ AB.BSA buffer: $\mathrm{AB}+1 \mathrm{mg} / \mathrm{mL}$ BSA. $1 \times$ AB.BSA.CAM: $1 \times$ AB.BSA +9 $\mu \mathrm{M}$ calmodulin. $1 \times$ G-buffer, $4 \mathrm{mM}$ Tris-Cl $(\mathrm{pH} 8.0$ ), and $0.2 \mathrm{mM}$ Calcium Chloride. $15 \times$ HKEM: 150 mM Hepes, $750 \mathrm{mM} \mathrm{KCl}, 75 \mathrm{mM} \mathrm{MgCl} 2,15 \mathrm{mM}$ EGTA.Na. Go mix: 1/25× F-actin, $0.05 \mathrm{mg} / \mathrm{mL}$ glucose oxidase, $0.01 \mathrm{mg} / \mathrm{mL}$ catalase, $1.5 \mathrm{mg} / \mathrm{mL}$ glucose, and $2 \mathrm{mM}$ of ATP in $1 \times$ AB.BSA.CAM.

myosin VI expression in Sf9 cells. Recombinant myosin VI was cloned into pBiEX-1 plasmid (Novagen). The construct contains Homo sapiens myosin VI, an N-terminal FLAG tag for purification by anti-FLAG resin, 6xHis tag for alternative purification using Ni-NTA resin, and a SNAP tag for optional attachment of benzylguanine (BG)-oligo. This construct was transfected into sf9 insect cells using transient transfection system (Escort IV, Sigma). The protein was expressed for $48-72$ hours at $27^{\circ} \mathrm{C}$ with constant shaking at $150 \mathrm{rpm}$. The expressed myosin VI is affinity purified using Anti-FLAG resin . The BG-oligo sequence is 5'-AAAAAAAAAAAAAAAAAAAA-3' with Cy5 modification at the 5'. The optional BG-oligo attachment does not change the gliding speed of actin (data not shown).

Actin Filament (F-actin) Polymerization. Rabbit skeletal muscle actin (Cytoskeleton, Inc; ) and rhodamine-labelled actin (Cytoskeleton, Inc; AR05) monomers were mixed at the ratio of 4 to 1 at a final concentration of $30 \mu \mathrm{M}$. Then, $4 \mu \mathrm{L}$ of the monomer mix was slowly pipetted into actin polymerization buffer which was prepared by mixing $30 \mu \mathrm{L}$ of Premix ( $3 \times$ HKEM, $30 \mathrm{mM}$ DTT, and $8 \mathrm{mM} \mathrm{ATP}), 6 \mu \mathrm{L}$ of $1 \times$ HKEM, and $51 \mu \mathrm{L} 1 \times$ G-buffer in a microcentrifuge tube. The tube was incubated on ice for $1 \mathrm{~h}$, then $1 \mu \mathrm{L}$ of $10 \mathrm{mM}$ phalloidin was added to the mix. The $\mathrm{F}$-actin was stored at $4^{\circ} \mathrm{C}$ for up to 2 months.

Coverslip Preparation. Corning D263 glass coverslips $(22 \mathrm{~mm} \times 50 \mathrm{~mm})$ were washed with isopropanol and then rinsed with deionized water before drying with nitrogen gas. After washing and drying, the coverslips were plasma-cleaned for 2 min at high setting (Harrick Plasma; PDC-32G) to remove impurities and organic residues. For nitrocellulose coating: coverslips were dipped in 1\% nitrocellulose in amyl acetate for 2 sec. Nitrocellulose solution was prepared by diluting 2\% nitrocellulose (Electron Microscopy Sciences; 12620-10) 
with amyl acetate (Electron Microscopy Sciences; 10815). Excess nitrocellulose was blotted from the corner of the coverslip with delicate task wipes before drying overnight in a vertical position. For HMDS coating: immediately after plasma cleaning, the coverslips were placed inside a vacuum desiccator together with a vial containing $300 \mu \mathrm{L}$ of HMDS solution at room temperature. Then, the desiccator was connected to a vacuum pump in a fume hood to deposit the HMDS vapor for $20 \mathrm{~min}$.

Coverslip Contact Angle Measurements. The hydrophobicity of treated and nontreated glass surfaces were characterized by contact angle measurements using a Krüss advance goniometer. De-ionized water droplets $(1 \mu \mathrm{L})$ were dispensed by an EasyDrop syringe pump system. After the droplet dispensation, an ellipse fit was used to obtain the contact angle of the water droplet at $20^{\circ} \mathrm{C}$. Droplet profiles were captured and their angles were analyzed by the in-built Krüss advance program version 1.11.0.15801. The angle of each replicate was the mean value of the left-most and right-most angle of each droplet at random locations on the coverslip.

Autofluorescence measurements. The epifluorescence images were obtained with an Oxford NanoImaging (ONi) microscope at 10\% laser power $(0.7-0.8 \mathrm{~mW})$, with $532 \mathrm{~nm}$ excitation, and $100 \mathrm{~ms}$ exposure. 2-min movies were taken. Autofluorescence of the samples are measured as mean pixel intensity (AU) of $400 \times$ 400 pixel $^{2}\left(46.68 \times 46.68 \mu \mathrm{m}^{2}\right)$ areas in the center of the image using ImageJ tools. ${ }^{31}$

Gliding Assay and Data Collection. Flow cell was made by sandwiching double-sided tape (Kapton) between the coverslips. A rectangle is cut on the double-sided tape with Graphtech cutting plotter. We estimate the volume of the flow cell to be $10 \mu \mathrm{L}$.

Gliding assay was performed by flowing $10 \mu \mathrm{L}$ of myosin VI into the flow cell followed by $10 \mathrm{~min}$ incubation to allow binding of myosin VI to the treated surface. Excess myosin was washed 3 times with $1 \times$ AB.BSA.CAM. Go mix containing 1/25X F-actin, $0.05 \mathrm{mg} / \mathrm{mL}$ glucose oxidase, $0.01 \mathrm{mg} / \mathrm{mL}$ catalase, $1.5 \mathrm{mg} / \mathrm{mL}$ glucose, and $2 \mathrm{mM}$ of ATP in $1 \times$ AB.BSA.CAM was introduced to the flow chamber. Then, imaging was immediately performed with ONi microscope (5\% power, excitation of a $1 \mathrm{~W} 532 \mathrm{~nm}$ laser, and a illumination angle of $54^{\circ}$ ). Movies of gliding actin filaments were obtained at $>10$ frames/sec.

Actin Gliding Speed Analysis. The gliding assay movies were analyzed with ImageJ. ${ }^{31}$ The movements of actin filaments were tracked with MTrackJ ${ }^{32}$ plugin based on the movement of their plus (pointed) end between consecutive frames (1 sec intervals) for at least 15 frames. Gliding speed of each filament was measured by dividing the total distance traveled by the elapsed time $(\Delta t=15-25$ sec $)$ to cover the measured distance.

Atomic Force Microscopy. All AFM images were acquired using a Dimension FastScan Bio (Bruker) using the "short and fat", or "long and thin" ScanAsyst-IN AIR or ScanAsyst-FLUID+ cantilever of an SNL probe ("sharp nitride lever", Bruker) in ScanAsyst Air or Fluid mode. NanoScope Analysis 2.0 and Mathematical (Wolfram Research) were used to measure the surface height distribution, HMDS film thickness, and generate the AFM image with appropriate color scales.

Capillary Tube Preparation. Rectangle Boro Capillary Tubes $(0.2 \mathrm{~mm} \times 2 \mathrm{~mm} \times 50 \mathrm{~mm}$; Vitrotubes (product number: 3520) were washed with isopropanol and then rinsed with deionized water before drying with a nitrogen gas. For nitrocellulose and HMDS treatments, the capillary tubes were plasma cleaned for 5 min at high RF setting (Harrick Plasma; Plasma Cleaner PDC-32G). For nitrocellulose coating: one end of the capillary tube was dipped into a microcentrifuge tube containing $30 \mu \mathrm{L} 1 \%$ nitrocellulose in amyl acetate until the liquid reached the other end. The excess nitrocellulose was drained by blotting one end with an absorbent task wipe, then hung vertically to dry overnight. For HMDS coating: The capillary tubes were placed vertically inside a vacuum desiccator chamber together with a vial containing $300 \mu \mathrm{L}$ of HMDS solution at room temperature. Then, the chamber was connected to a vacuum pump in a fume hood to deposit the HMDS vapor for $20 \mathrm{~min}$. 
Capillary Tube Contact Angle Measurements. The capillary tubes were arranged in a clear flat bottom tray with multiple wells and stood upright. A Canon EOS 77D camera body with an EF-S 35mm f/2.8 Macro IS STM Canon lens was arranged reasonably far away to minimize camera distortion effects but close enough to capture the 4 tubes such that the meniscus and bottom of the capillary tube were clearly visible. $70 \mu \mathrm{L}$ of a solution of $1 \mathrm{~mL}$ MilliQ water and $\sim 5 \mu \mathrm{L}$ of red food coloring was loaded into each well that contained a capillary tube. The capillary tubes were left undisturbed for 20 min after which a still photo was taken. The photo was analyzed using Logger Pro 3 by Vernier. The height of each capillary tube was measured to be $50.9 \mathrm{~mm}$ and was used to set the scale for each height measurement. The height from the base of the capillary tube to the lower meniscus was recorded as the height. 3 repeats were taken with freshly prepared tubes each time. Jurin's Law was used to calculate the contact angles, first using the assumption that the "radius" of the capillary tube was circular

$$
r \sim \sqrt{\frac{l \times w}{\pi}}=\sqrt{\frac{0.2 \mathrm{~mm} \times 2 \mathrm{~mm}}{\pi}},
$$

then using the assumption that the capillary tube was a pair of parallel plates $(\mathrm{r}=0.2 \mathrm{~mm})$. Jurin's law is unable to calculate the contact angle when the water height is the full length of the capillary tube which happens consistently when the capillary tube is plasma cleaned.

Gliding Assay with Capillary Tubes. The volume of a capillary tube is $200 \mu \mathrm{L}$ according to the specifications given by the manufacturer. $200 \mu \mathrm{L}$ of myosin VI is flowed into the capillary tube by dipping one end into the solution and tipping the tube such that it is horizontal. This allows the solution to be taken up via capillary effect. The myosin VI is allowed to incubate for $15 \mathrm{~min}$. In order to flow the next few solutions, a different approach must be used. The capillary tube is placed in the solution and a dry wipe is used to absorb the solution from the other end causing the capillary tube to take in the solution it is placed in. Using this method, we washed the capillary tube with approximately $600 \mu \mathrm{L}$ of $1 \times \mathrm{AB}$.BSA.CAM followed by $200 \mu \mathrm{L}$ of Go-mixture containing $1 / 25 \times$ F-actin, $0.05 \mathrm{mg} / \mathrm{mL}$ glucose oxidase, $0.01 \mathrm{mg} / \mathrm{mL}$ catalase, $1.5 \mathrm{mg} / \mathrm{mL}$ glucose, and $2 \mathrm{mM}$ of ATP in $1 \mathrm{X}$ AB.BSA.CAM. Then, imaging was immediately performed with ONi microscope ( $8 \%$ power, excitation of $532 \mathrm{~nm}$ or green laser, and a TIRF excitation angle of $\sim 50^{\circ}$ ). In most experiments, 2-min movies of gliding actin filaments were obtained at $10 \mathrm{fps}$.

Statistical analysis. The $p$-values were computed by two-tail unpaired Student's t test using Prism 9 (GraphPad). We calculated the error bars as mean \pm SD throughout this work, with the exception of Fig. 4B $($ mean $\pm \mathrm{SEM})$. 


\title{
Supporting Information
}

\section{A simple surface modification to generate atomically-flat and hydrophobic substrates for gliding assays with protein motors}

\begin{abstract}
Isadonna F. Tengganu ${ }^{a}$, Neil Karerakattil ${ }^{\mathrm{b}}$, Swarup Dey ${ }^{\mathrm{a}, \mathrm{c}}$, Devika Kishnan ${ }^{\mathrm{d}}$, and Rizal F. Hariadi ${ }^{(\mathbb{D})}$ a,b,1
aBiodesign Center for Molecular Design and Biomimetics (at the Biodesign Institute) at Arizona State University, Tempe, AZ 85287, USA.; ${ }^{b}$ Department of Physics, Arizona State University, Tempe, AZ 85287, USA.; ' School of Molecular Sciences, Arizona State University, Tempe, AZ 85287, USA.; ${ }^{\mathrm{d}}$ School of Biological and Health System Engineering, Arizona State University, Tempe, AZ 85287, USA.
\end{abstract}

${ }^{1}$ To whom correspondence should be addressed. E-mail: rhariadi@asu.edu

\section{Contents}

S1 Supporting Information $\quad 2$

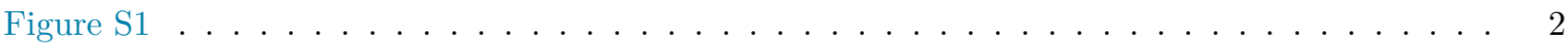

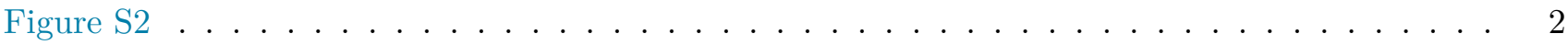

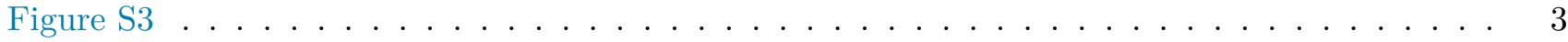

S2 Supporting Movies $\quad 3$

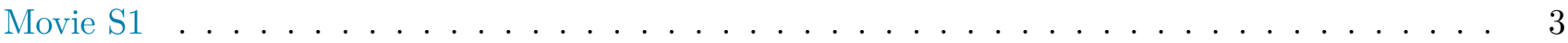

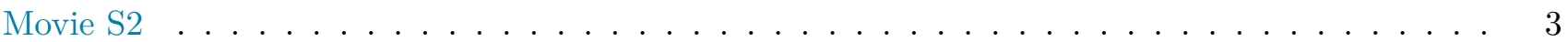

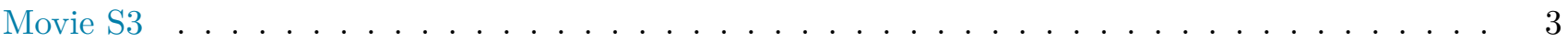

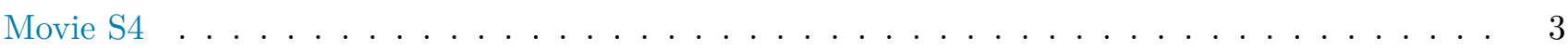

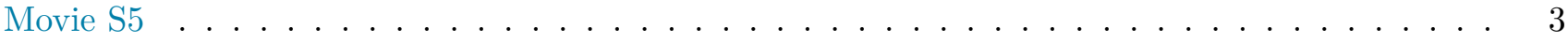

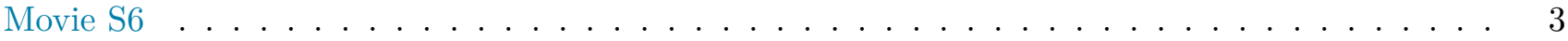

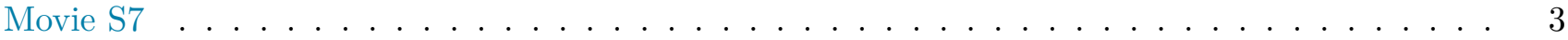

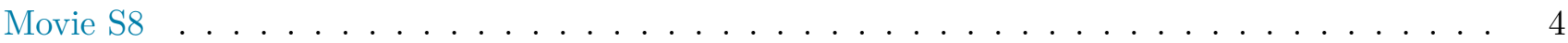

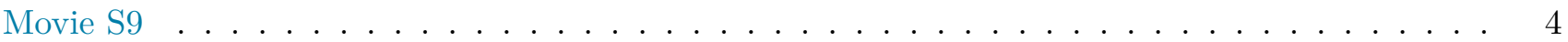

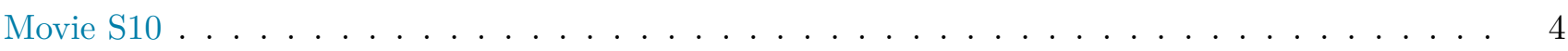

Movie $\mathrm{S} 11 \ldots \ldots \ldots \ldots \ldots \ldots \ldots \ldots \ldots \ldots$

Movie $\mathrm{S} 12 \ldots \ldots \ldots \ldots \ldots \ldots \ldots \ldots \ldots \ldots \ldots \ldots \ldots$

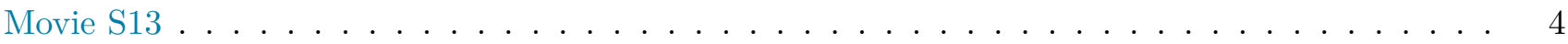




\section{S1. Supporting Information}

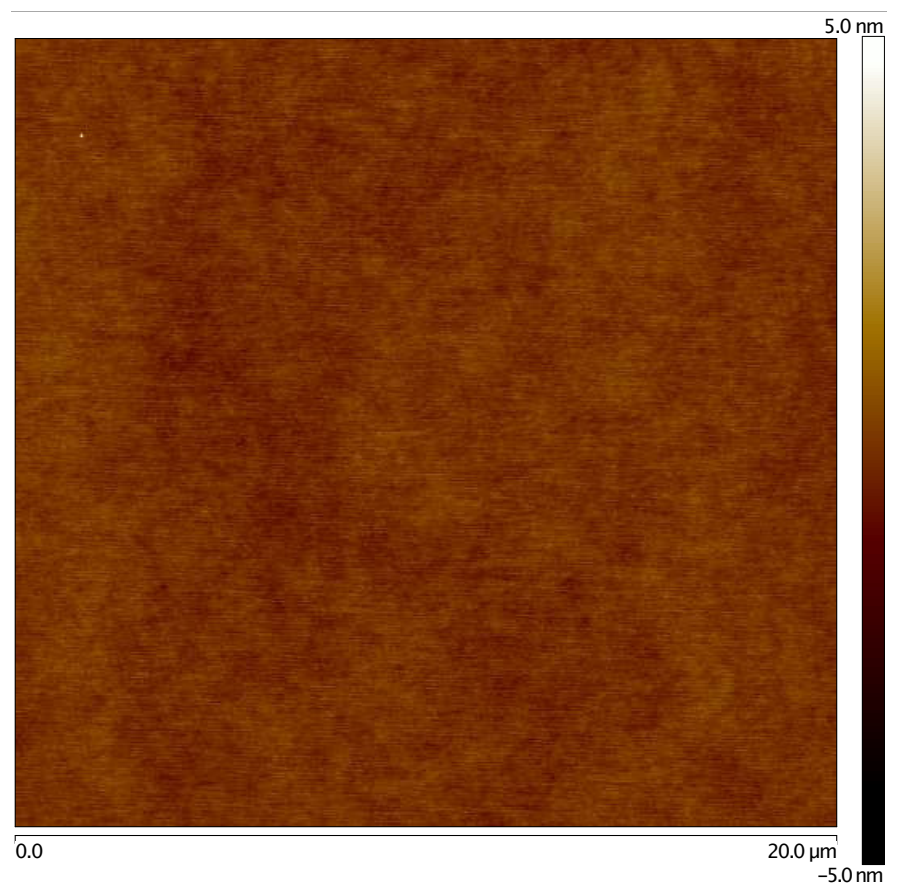

Fig. S1 AFM image of HMDS-coated glass surface. Vapor deposition of HMDS maintains the surface flatness.

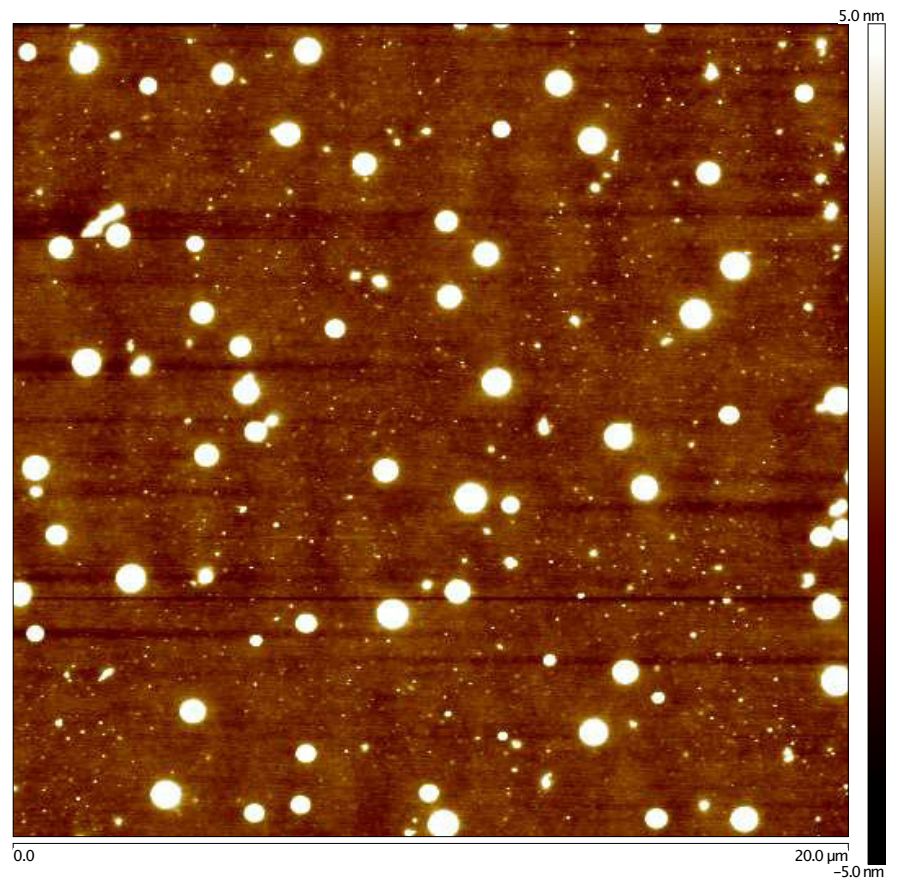

Fig. S2 AFM image of nitrocellulose-coated glass surface shows that nitrocellulose coating does not produce a flat surface. 


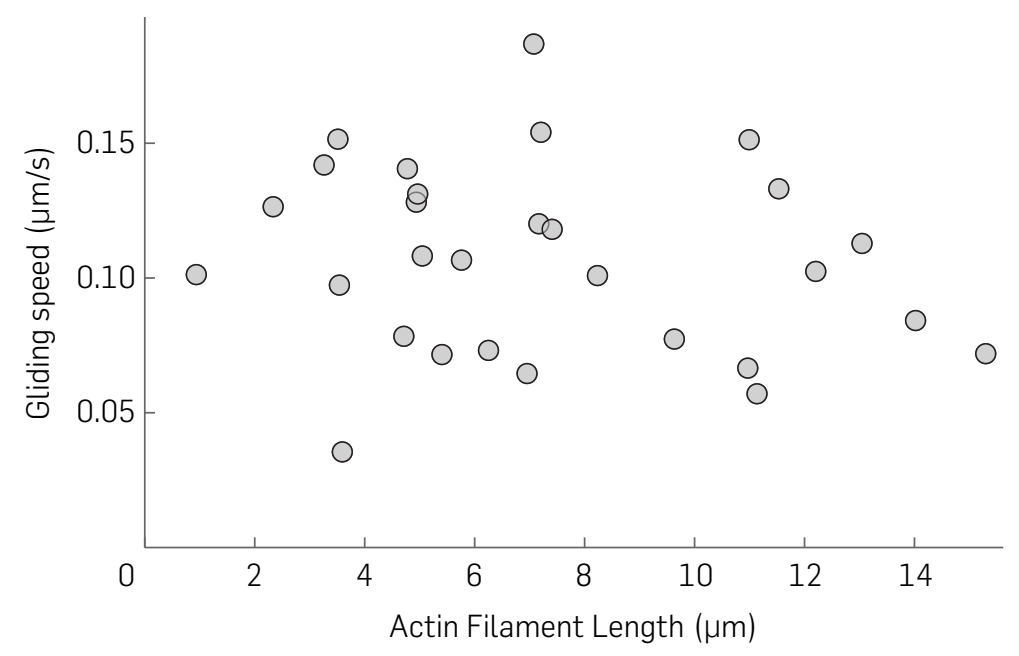

Fig. S3 Gliding speed of actin filaments in capillary tubes ( $N=29$ filaments). One data point (296 $\mathrm{nm} / \mathrm{sec})$ was excluded from the plot and from calculating the mean \pm SD. This designated outlier is $>5 \sigma$ from the estimated mean \pm SD $(107 \pm 35 \mathrm{~nm} / \mathrm{sec})$. The mean was calculated after excluding the outlier.

\section{S2. Supporting Movies}

Movies S1-S13 are available at https://github.com/bionicslab/HMDS-manuscript.

Movie S1 Photobleaching of glass coverslip.

Movie S2 Photobleaching of HMDS-coated coverslip.

Movie S3 Photobleaching of nitrocellulose-coated coverslip.

Movie S4 Gliding assay of actin filaments on myosin VI immobilized on HMDS-coated surface (PC glass + HMDS).

Movie S5 Gliding assay of actin filaments on myosin VI immobilized on nitrocellulose-coated surface (PC glass + nitrocellulose).

Movie S6 Gliding assay of actin filaments on myosin VI immobilized on HMDS-coated surface preincubated with BSA (PC glass + HMDS + BSA).

Movie S7 Gliding assay of actin filaments on myosin VI immobilized on plasma-cleaned glass preincubated with BSA (PC glass + BSA). 
Movie S8 Gliding assay of actin filaments on myosin VI immobilized on HMDS-coated surface preincubated with Tween-20 (PC glass + HMDS + Tween-20).

Movie S9 Gliding assay of actin filaments on myosin VI immobilized on plasma-cleaned glass preincubated with Tween-20 (PC glass + Tween-20).

Movie S10 Gliding assay of actin filaments on myosin VI immobilized on plasma-cleaned glass (PC glass). Movie S11 Gliding assay of actin filaments on myosin VI immobilized on HMDS-coated capillary tube (PC glass + HMDS).

Movie S12 Gliding assay of actin filaments on myosin VI immobilized on untreated capillary tube.

Movie S13 Gliding assay of actin filaments on myosin VI immobilized on plasma-cleaned capillary tube (PC glass). 